\title{
Optimal Design and Reduced Threshold in Vertically Emitting Circular Bragg Disk Resonator Lasers
}

\author{
Xiankai Sun, Jacob Scheuer, Senior Member, IEEE, and Amnon Yariv, Life Fellow, IEEE
}

\begin{abstract}
We derive a comprehensive coupled-mode theory, including resonant vertical emission effects, for the analysis of nonperiodic circular Bragg lasers. We derive the governing characteristic equation for such lasers, yielding the threshold gain level and the resonance frequency. By reducing the threshold gain and maximizing the ratio of "useful signal" to the power leakage, we find optimum conditions for vertically emitting circular Bragg micordisk lasers which indicate that low-threshold operation is possible.
\end{abstract}

Index Terms-Bragg resonators, integrated optics, photonic crystals, surface emitting lasers.

\section{INTRODUCTION}

I $\mathrm{N}$ the past few years, circular optical resonators have been receiving increasing attention, primarily because of their applications in optical communication systems [1]-[4], cavity quantum electrodynamics [5], and biochemical sensing [6]. Such resonators are becoming essential building blocks for future photonic devices and applications.

For many of these applications, both a high-quality factor $(Q)$ and a small modal volume are desirable. The $Q$ defined as the number of optical periods to decay by $e^{-1}$, is a figure of merit describing the energy storage capacity of the resonator. The modal volume, which characterizes the interaction region of photons and emitters, is of particular importance in the realization of compact low-threshold lasers in integrated optical systems.

In conventional circular resonators, employing the total internal reflection (TIR) mechanism for the confinement of light, achieving both high $Q$ and small modal volume is difficult. Small modal volume requires small circumference and bending radius, which results in large power dissipation due to "bending loss" [7], thus, decreasing the $Q$. This linkage can be broken by confining light using Bragg reflection [8], [9]. Such devices, known as annular Bragg resonators (ABRs), are designed to confine the light in a central disk or in a radial defect region by radial Bragg reflection.

For the important application of optical resonators in laser devices, optimal light confinement is crucial for decreasing the

Manuscript received August 24, 2006; revised February 7, 2007. This work was supported in part by the Defense Advanced Research Projects Agency (DARPA) and in part by the National Science Foundation.

$\mathrm{X}$. Sun is with the Department of Applied Physics, California Institute of Technology, Pasadena, CA 91125 USA (e-mail: xksun@caltech.edu).

J. Scheuer is with the School of Electrical Engineering and the Center for Nanosciences and Nanotechnology, Tel-Aviv University, Ramat-Aviv, Tel-Aviv 69978, Israel (e-mail: kobys@eng.tau.ac.il).

A. Yariv is with the Department of Applied Physics and also with the Department of Electrical Engineering, California Institute of Technology, Pasadena, CA 91125 USA (e-mail: ayariv@caltech.edu).

Digital Object Identifier 10.1109/JSTQE.2007.893559

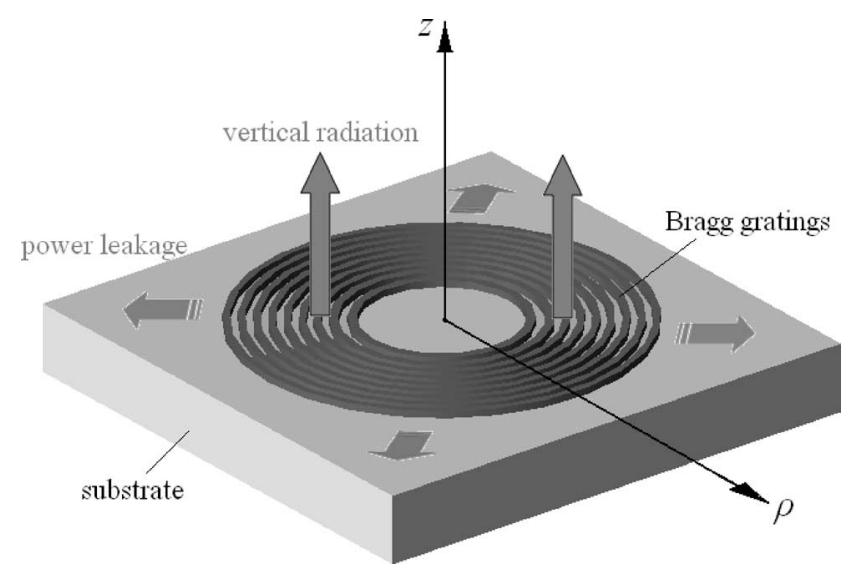

Fig. 1. Illustration of a circular Bragg microdisk laser.

lasing threshold [10], [11]. Although optimal confinement can be achieved using a 3-D photonic bandgap structure, the fabrication of such structure is not simple and issues of in- and out-coupling are yet unsolved. In a 2-D geometry, an annular Bragg resonator can provide a complete 2-D feedback, and strongly localize the light in the predesigned region. In addition, the annular Bragg gratings can also serve as an output coupler to diffract the light out of the plane via first-order diffraction, producing a circular laser beam with narrow divergence.

The properties of passive ABR structures have been studied theoretically [9], yielding the optimal Bragg layer structure for high $Q$ and small modal volume. Recently, we have realized ABR-based lasers in InGaAsP semiconductor materials [12] exhibiting low threshold [13] and small modal volume [11]. However, a comprehensive theoretical analysis of active ABRs (i.e., lasers) and optimization of their threshold level and efficiency are yet to be done.

In this paper, we analyze theoretically the threshold levels and modal properties of circular Bragg lasers employing mixedorder Bragg gratings to achieve both confinement and efficient vertical emission [9]. The paper is organized as follows. In Section II, we derive the coupled-mode theory. In Section III, we apply the coupled-mode theory to the analysis of circular Bragg microdisk lasers. In Section IV, we present the numerical results, based on which we suggest an optimal design for the microdisk lasers, and in Section V we present the conclusions.

\section{BASIC Formulation By COUPLED-MODE THEORY}

As illustrated in Fig. 1, a microdisk Bragg resonator consists of a central disk of dielectric material surrounded by radial perturbation of Bragg gratings. In such a structure, the 
electromagnetic fields can be categorized into TE and TM polarizations. When the perturbation $\Delta n^{2}(\rho, z)$ is scalar, it does not couple the orthogonal polarizations and, therefore, each case can be analyzed separately. Thus, in our analysis, we focus on the TE-polarized field while the analysis of the TM polarization is similar. All the field components can be obtained from the $z$-component of the electric field satisfying the Helmholtz equation

$$
\left[\frac{1}{\rho} \frac{\partial}{\partial \rho}\left(\rho \frac{\partial}{\partial \rho}\right)+\frac{1}{\rho^{2}} \frac{\partial^{2}}{\partial \varphi^{2}}+k_{0}^{2} n^{2}(\rho, z)+\frac{\partial^{2}}{\partial z^{2}}\right] E_{z}(\rho, \varphi, z)=0
$$

where

$$
k_{0}=\frac{\omega}{c}=\frac{2 \pi}{\lambda_{0}}
$$

is the wave number in vacuum.

For an azimuthally propagating electromagnetic mode, the azimuthal dependence can be separated and the $E_{z}$ can be expressed as

$$
E_{z}(\rho, \varphi, z)=E_{z}^{(m)}(\rho, z) \exp (i m \varphi)
$$

where $m$ is the azimuthal mode number. Introducing (2) into (1) yields

$\frac{1}{\rho} \frac{\partial}{\partial \rho}\left(\rho \frac{\partial E_{z}^{(m)}}{\partial \rho}\right)+\left[-\frac{m^{2}}{\rho^{2}}+k_{0}^{2} n^{2}(\rho, z)+\frac{\partial^{2}}{\partial z^{2}}\right] E_{z}^{(m)}=0$.

Assuming that the vertical and transverse dependences of the field can be separated, we express the field as $E_{z}^{(m)}(\rho, z)=$ $R_{m}(\rho) Z(z)$ where $Z(z)$ is the fundamental mode profile of the planar slab waveguide satisfying

$$
\left(k_{0}^{2} \varepsilon_{r}(z)+\frac{\partial^{2}}{\partial z^{2}}\right) Z(z)=\beta^{2} Z(z)
$$

and $\beta=k_{0} n_{\text {eff }}$ is the in-plane propagation constant.

Within the unperturbed region, the dielectric constant has the form of $n^{2}(\rho, z)=\varepsilon_{r}(z)+i \varepsilon_{i}(z)$, where $\left|\varepsilon_{i}(z)\right| \ll \varepsilon_{r}(z)$ represents the gain/loss coefficient. Substituting $n^{2}$ into (3) yields

$$
\frac{1}{\rho} \frac{d}{d \rho}\left(\rho \frac{d R_{m}}{d \rho}\right)+\left[\beta^{2}+i k_{0}^{2} \varepsilon_{i}-\frac{m^{2}}{\rho^{2}}\right] R_{m}=0 .
$$

Introducing the normalized radius $x=\beta \rho$ into (5) yields

$$
\frac{1}{x} \frac{d}{d x}\left(x \frac{d R_{m}}{d x}\right)+\left(1+i \frac{k_{0}^{2} \varepsilon_{i}}{\beta^{2}}-\frac{m^{2}}{x^{2}}\right) R_{m}=0 .
$$

The modal solutions of (6) can be written as

$$
R_{m}(x)=A(x) H_{m}^{(1)}(x)+B(x) H_{m}^{(2)}(x)
$$

and

$$
E_{z}^{(m)}(x, z)=\left[A(x) H_{m}^{(1)}(x)+B(x) H_{m}^{(2)}(x)\right] Z(z) .
$$

Within the perturbed region, the dielectric constant includes an additional term $n^{2}(\rho, z)=\varepsilon_{r}(z)+i \varepsilon_{i}(z)+\Delta \varepsilon(\rho, z)$. In [8], we showed that for optimal field confinement, the perturbation $\Delta \varepsilon(\rho, z)$ could be expanded in Hankel-phased plane-wave series. Assuming small perturbations, we keep only the first two terms in the expansion (since $\left|\varepsilon_{i}(z)\right| \ll \varepsilon_{r}(z)$, we consider only index modulation).

$$
\begin{aligned}
\Delta & \varepsilon(\rho, z) \\
= & -\Delta \varepsilon_{0}\left[\sum_{l= \pm 1, \pm 2} a_{l}(z) \exp \left(-i l \Phi\left(H_{m}^{(1)}\left(\beta_{\text {design }} \rho\right)\right)\right)\right] \\
= & -\Delta \varepsilon_{0}\left[\sum_{l= \pm 1, \pm 2} a_{l}(z) \exp \left(-i l \Phi\left(H_{m}^{(1)}(x)\right)\right) \exp (-i l \delta x)\right] \\
= & -\Delta \varepsilon_{0}\left[a_{2}(z) \exp \left(-2 i \Phi\left(H_{m}^{(1)}(x)\right)\right) \exp (-2 i \delta x)\right. \\
& +a_{-2}(z) \exp \left(2 i \Phi\left(H_{m}^{(1)}(x)\right)\right) \exp (2 i \delta x) \\
& +a_{1}(z) \exp \left(-i \Phi\left(H_{m}^{(1)}(x)\right)\right) \exp (-i \delta x) \\
& \left.+a_{-1}(z) \exp \left(i \Phi\left(H_{m}^{(1)}(x)\right)\right) \exp (i \delta x)\right] \\
= & -\Delta \varepsilon_{0}\left[a_{2}(z) \frac{H_{m}^{(2)}}{H_{m}^{(1)}} e^{-2 i \delta x}+a_{-2}(z) \frac{H_{m}^{(1)}}{H_{m}^{(2)}} e^{2 i \delta x}\right. \\
& \left.+a_{1}(z) \frac{H_{m}^{(2)}}{\left|H_{m}^{(1)}\right|} e^{-i \delta x}+a_{-1}(z) \frac{H_{m}^{(1)}}{\left|H_{m}^{(1)}\right|} e^{i \delta x}\right]
\end{aligned}
$$

$\delta \equiv \frac{\beta_{\text {design }}-\beta}{\beta},|\delta| \ll 1$

is the normalized frequency detuning factor, representing the relative frequency shift from the optimal coupling design implying that $\beta_{\text {design }} \rho=x(1+\delta)$. For simplicity, we approximate $H_{m}^{(1,2)}(x(1+\delta))$ by $H_{m}^{(1,2)}(x) e^{ \pm i \delta x}[8]$. Note that, we have intentionally dropped the zeroth order term from (8) because it can be incorporated into the unperturbed part of $\varepsilon_{r}(z)$. In addition, it should be noted that the coefficients $a_{l}(z)$ are nonvanishing only for $z$ located in the grating layers. These coefficients are directly related to the radiation coupling between in-plane waves and the vertically radiating waves, and to the feedback coupling between the outgoing and the incoming in-plane waves.

To account for the vertically radiating fields, we introduce an additional term $\Delta E(x, z)$ to $E_{z}$

$$
\begin{aligned}
E_{z}^{(m)}(x, z) & =\tilde{R}_{m}(x) Z(z)+\Delta E(x, z) \\
& =\left[\tilde{A}(x) H_{m}^{(1)}(x)+\tilde{B}(x) H_{m}^{(2)}(x)\right] Z(z)+\Delta E(x, z)
\end{aligned}
$$

The tildes on the field amplitudes are used to distinguish them from those in (7). We assume that the radiating field $\Delta E(x, z)$ has an $\exp \left( \pm i k_{0} z\right)$ dependence on $z$ in free space, i.e.

$$
\left[\frac{1}{\rho} \frac{\partial}{\partial \rho}\left(\rho \frac{\partial}{\partial \rho}\right)-\frac{m^{2}}{\rho^{2}}\right] \Delta E=0 .
$$


Substituting (9a) into (3) yields

$$
\begin{gathered}
\frac{Z}{x} \frac{d}{d x}\left(x \frac{d \tilde{R}_{m}}{d x}\right)+\left[1+\frac{i k_{0}^{2} \varepsilon_{i}(z)+k_{0}^{2} \Delta \varepsilon(x, z)}{\beta^{2}}-\frac{m^{2}}{x^{2}}\right] \tilde{R}_{m} Z \\
+\frac{1}{\beta^{2}}\left(k_{0}^{2} \varepsilon_{r}(z)+i k_{0}^{2} \varepsilon_{i}(z)+k_{0}^{2} \Delta \varepsilon(x, z)+\frac{\partial^{2}}{\partial z^{2}}\right) \Delta E=0 .
\end{gathered}
$$

Introducing (8) and (9b) into (11) and neglecting the second derivatives of $A(x)$ and $B(x)$ (slowly varying envelope approximation) leads to

$$
\begin{aligned}
& Z\left(2 \frac{d A}{d x} \frac{d H_{m}^{(1)}}{d x}+A \frac{d^{2} H_{m}^{(1)}}{d x^{2}}+2 \frac{d B}{d x} \frac{d H_{m}^{(2)}}{d x}+B \frac{d^{2} H_{m}^{(2)}}{d x^{2}}\right) \\
& +\frac{Z}{x}\left(\frac{d A}{d x} H_{m}^{(1)}+A \frac{d H_{m}^{(1)}}{d x}+\frac{d B}{d x} H_{m}^{(2)}+B \frac{d H_{m}^{(2)}}{d x}\right) \\
& +\left(1+i \frac{k_{0}^{2} \varepsilon_{i}}{\beta^{2}}-\frac{m^{2}}{x^{2}}\right)\left(A H_{m}^{(1)} Z+B H_{m}^{(2)} Z\right) \\
& +\frac{1}{\beta^{2}}\left(k_{0}^{2} \varepsilon_{r}+i k_{0}^{2} \varepsilon_{i}+\frac{\partial^{2}}{\partial z^{2}}\right) \Delta E \\
& =\frac{k_{0}^{2} \Delta \varepsilon_{0}}{\beta^{2}}\left(a_{2} \frac{H_{m}^{(2)}}{H_{m}^{(1)}} e^{-2 i \delta x}+a_{-2} \frac{H_{m}^{(1)}}{H_{m}^{(2)}} e^{2 i \delta x}\right. \\
& \left.+a_{1} \frac{H_{m}^{(2)}}{\left|H_{m}^{(1)}\right|} e^{-i \delta x}+a_{-1} \frac{H_{m}^{(1)}}{\left|H_{m}^{(1)}\right|} e^{i \delta x}\right) \\
& \quad \times\left(A H_{m}^{(1)} Z+B H_{m}^{(2)} Z+\Delta E\right) .
\end{aligned}
$$

Using the modal solution in the unperturbed region

$$
\begin{gathered}
\left(A \frac{d^{2} H_{m}^{(1)}}{d x^{2}}+B \frac{d^{2} H_{m}^{(2)}}{d x^{2}}\right)+\frac{1}{x}\left(A \frac{d H_{m}^{(1)}}{d x}+B \frac{d H_{m}^{(2)}}{d x}\right) \\
+\left(1-\frac{m^{2}}{x^{2}}\right)\left(A H_{m}^{(1)}+B H_{m}^{(2)}\right)=0
\end{gathered}
$$

and the approximations

$$
\left\{\begin{array}{l}
\frac{H_{m}^{(1,2)}(x)}{x} \ll \frac{d H_{m}^{(1,2)}(x)}{d x} \\
\frac{d^{n} H_{m}^{(1,2)}(x)}{d x^{n}} \approx( \pm i)^{n} H_{m}^{(1,2)}(x)
\end{array}\right.
$$

for large $x$ radius (see [8]), we find that by inserting (13) and (14) into (12), we get

$$
\begin{aligned}
& Z\left(2 i \frac{d A}{d x} H_{m}^{(1)}-2 i \frac{d B}{d x} H_{m}^{(2)}\right)+i \frac{k_{0}^{2} \varepsilon_{i}}{\beta^{2}}\left(A H_{m}^{(1)} Z+B H_{m}^{(2)} Z\right) \\
& +\frac{1}{\beta^{2}}\left(k_{0}^{2} \varepsilon_{r}+i k_{0}^{2} \varepsilon_{i}+\frac{\partial^{2}}{\partial z^{2}}\right) \Delta E \\
& =\frac{k_{0}^{2} \Delta \varepsilon_{0}}{\beta^{2}}\left(a_{2} \frac{H_{m}^{(2)}}{H_{m}^{(1)}} e^{-2 i \delta x}+a_{-2} \frac{H_{m}^{(1)}}{H_{m}^{(2)}} e^{2 i \delta x}\right.
\end{aligned}
$$

$$
\begin{aligned}
& \left.+a_{1} \frac{H_{m}^{(2)}}{\left|H_{m}^{(1)}\right|} e^{-i \delta x}+a_{-1} \frac{H_{m}^{(1)}}{\left|H_{m}^{(1)}\right|} e^{i \delta x}\right) \\
& \times\left(A H_{m}^{(1)} Z+B H_{m}^{(2)} Z+\Delta E\right) .
\end{aligned}
$$

The right-hand side of (15) can be regarded as source terms that drive the outgoing and incoming waves on the left-hand side. From the phase match condition, both the source and wave must have close phase dependence. Grouping terms with the same kind of Hankel functions leads to a set of coupled equations in $A(x), B(x)$, and $\Delta E(x, z)$ given by

$$
\begin{aligned}
& 2 i \frac{d A}{d x} H_{m}^{(1)} Z+i \frac{k_{0}^{2} \varepsilon_{i}}{\beta^{2}} A H_{m}^{(1)} Z \\
& \quad=\frac{k_{0}^{2} \Delta \varepsilon_{0}}{\beta^{2}}\left[a_{-2} B H_{m}^{(1)} e^{2 i \delta x} Z+a_{-1} \frac{\Delta E}{\left|H_{m}^{(1)}\right|} H_{m}^{(1)} e^{i \delta x}\right] \\
& -2 i \frac{d B}{d x} H_{m}^{(2)} Z+i \frac{k_{0}^{2} \varepsilon_{i}}{\beta^{2}} B H_{m}^{(2)} Z \\
& =\frac{k_{0}^{2} \Delta \varepsilon_{0}}{\beta^{2}}\left[a_{2} A H_{m}^{(2)} e^{-2 i \delta x} Z+a_{1} \frac{\Delta E}{\left|H_{m}^{(1)}\right|} H_{m}^{(2)} e^{-i \delta x}\right] \\
& \left(k_{0}^{2} \varepsilon_{r}+\frac{\partial^{2}}{\partial z^{2}}\right) \Delta E \\
& \quad=k_{0}^{2} \Delta \varepsilon_{0}\left(a_{1} A\left|H_{m}^{(1)}\right| e^{-i \delta x} Z+a_{-1} B\left|H_{m}^{(1)}\right| e^{i \delta x} Z\right) .
\end{aligned}
$$

Applying Green's function technique to (16c), $\Delta E$ can be expressed as

$$
\Delta E=\left(s_{1} A e^{-i \delta x}+s_{-1} B e^{i \delta x}\right)\left|H_{m}^{(1)}\right|
$$

where

$$
s_{l}(z)=k_{0}^{2} \Delta \varepsilon_{0} \int_{-\infty}^{+\infty} a_{l}\left(z^{\prime}\right) Z\left(z^{\prime}\right) G\left(z, z^{\prime}\right) d z^{\prime}
$$

and the Green's function $G\left(z, z^{\prime}\right)$ satisfies the following

$$
\left(k_{0}^{2} \varepsilon_{r}(z)+\frac{\partial^{2}}{\partial z^{2}}\right) G\left(z, z^{\prime}\right)=\delta\left(z-z^{\prime}\right) .
$$

Equation (17) indicates that the radial dependence of the radiated field is similar to that of the in-plane field. Substituting (17) into (16a) and (16b), multiplying both sides by $Z(z)$, and integrating over $z$ eliminates the $z$ dependence.

In the case of index grating, we can always choose $a_{l}$ such that $a_{-l}=a_{l}^{*}=a_{l}$, thus, we can define the normalization constant [14]

$$
P \equiv \int_{-\infty}^{+\infty} Z^{2}(z) d z=\frac{2 \mu_{0} \omega}{\beta}
$$


the gain coefficient

$$
g_{A} \equiv-\frac{k_{0}^{2}}{2 P \beta^{2}} \int_{-\infty}^{+\infty} \varepsilon_{i}(z) Z^{2}(z) d z
$$

the radiation coupling coefficient

$$
h_{1}=h_{ \pm 1, \pm 1} \equiv \frac{i k_{0}^{2} \Delta \varepsilon_{0}}{2 P \beta^{2}} \int_{-\infty}^{+\infty} a_{ \pm 1}(z) s_{ \pm 1}(z) Z(z) d z
$$

and the feedback coupling coefficient

$$
h_{2}=h_{ \pm 2} \equiv \frac{k_{0}^{2} \Delta \varepsilon_{0}}{2 P \beta^{2}} \int_{-\infty}^{+\infty} a_{ \pm 2}(z) Z^{2}(z) d z
$$

Note that $g_{A}, h_{1}$, and $h_{2}$ are dimensionless (already normalized by $\beta$ ).

Introducing these definitions, we get a set of evolution equations for the amplitudes of the outgoing and incoming waves in the resonator

$$
\left\{\begin{array}{l}
\frac{d A}{d x}=\left(g_{A}-h_{1}\right) A-\left(h_{1}+i h_{2}\right) B e^{2 i \delta x} \\
\frac{d B}{d x}=-\left(g_{A}-h_{1}\right) B+\left(h_{1}+i h_{2}\right) A e^{-2 i \delta x}
\end{array}\right.
$$

Let $u=g_{A}-h_{1}$ and $\nu=h_{1}+i h_{2}$, then

$$
\left\{\begin{array}{l}
\frac{d A}{d x}=u A-v B e^{2 i \delta x} \\
\frac{d B}{d x}=-u B+v A e^{-2 i \delta x}
\end{array}\right.
$$

The generic solution of (25) can be readily obtained as

$$
\left\{\begin{aligned}
A(x)= & {\left[C_{1} \exp (S x)+C_{2} \exp (-S x)\right] \exp (i \delta x) } \\
B(x)= & -\frac{1}{v}\left[C_{1}(S-u+i \delta) \exp (S x)\right. \\
& \left.-C_{2}(S+u-i \delta) \exp (-S x)\right] \exp (-i \delta x)
\end{aligned}\right.
$$

where $S \equiv \sqrt{(u-i \delta)^{2}-v^{2}}$.

Note that from (9) and (26), we may define, in analogy to the linear grating case, an effective radial propagation constant $\tilde{\beta}$, such that $i \Phi\left[H_{m}^{(1)}(\tilde{\beta} \rho)\right]=i \Phi\left[H_{m}^{(1)}(x)\right]+i \delta x \pm S x$. Invoking the large radius approximation $\Phi\left[H_{m}^{(1)}(x)\right]=x-(m \pi / 2)-$ $(\pi / 4)$ yields $i \tilde{\beta} \rho=i x+i \delta x \pm S x=i \beta_{\operatorname{design}} \rho \pm S \beta \rho$, giving $\tilde{\beta}=\beta_{\text {design }} \pm i S \beta$. A real $S$ indicates the existence of a bandgap in the dispersion relation, which reflects the waves in the grating region and allows the light to be confined in the central disk.

In the unperturbed region where $\Delta \varepsilon=0,(16)$ is reduced to

$$
\left\{\begin{array}{l}
\frac{d A}{d x}=-\frac{k_{0}^{2} \varepsilon_{i}}{2 \beta^{2}} A \\
\frac{d B}{d x}=\frac{k_{0}^{2} \varepsilon_{i}}{2 \beta^{2}} B
\end{array}\right.
$$

whose solution is

$$
\left\{\begin{array}{l}
A(x)=A(0) \exp \left(g_{A} x\right) \\
B(x)=B(0) \exp \left(-g_{A} x\right)
\end{array}\right.
$$

\section{Circular Bragg Microdisk Lasers}

While the light in the microdisk laser illustrated in Fig. 1 is designed to be confined primarily in the central disk region, it is also coherently coupled in the vertical direction (out of the resonator) and emitted from the surface due to the first-order Bragg diffraction in the grating region. Although it might seem that this scattering is a loss mechanism, it must be emphasized that this portion of the light is our useful signal. To solve the mode profile and characteristic equation we set the following boundary conditions:

1) At the center of the disk $x=0$, the total amplitude of each Fourier component of the field must be finite, requiring $A(0)=B(0)=A$. Therefore, the amplitudes and the electric field in the disk region are given by

$$
\left\{\begin{aligned}
A(x)=A \exp \left(g_{A} x\right) \\
B(x)=A \exp \left(-g_{A} x\right) \\
E_{z}^{(m)}(x, z)=A Z(z)\left(\exp \left(g_{A} x\right) H_{m}^{(1)}(x)\right. \\
\left.\quad+\exp \left(-g_{A} x\right) H_{m}^{(2)}(x)\right)
\end{aligned}\right.
$$

2) At the exterior boundary $x=x_{R}$, we assume that there is no incoming wave from $x \geq x_{R}$, and, therefore, we have $B\left(x_{R}\right)=0$, which yields $C_{2}=\frac{S-u+i \delta}{S+u-i \delta} \exp \left(2 S x_{R}\right) C_{1}$. The amplitudes and the electric field in the grating region are then given by (30) shown at the bottom of the page.

3) At the interface $x=x_{0}$, both the field and its first-order derivative must be continuous (for the TE polarization)

$$
\begin{aligned}
& A Z(z)\left(\exp \left(g_{A} x_{0}\right) H_{m}^{(1)}\left(x_{0}\right)+\exp \left(-g_{A} x_{0}\right) H_{m}^{(2)}\left(x_{0}\right)\right) \\
& =C_{1} \exp \left(S x_{0}+i \delta x_{0}\right)\left[1+\frac{S-u+i \delta}{S+u-i \delta} \exp \left(2 S\left(x_{R}-x_{0}\right)\right)\right] \\
& \quad \times\left(H_{m}^{(1)}\left(x_{0}\right) Z(z)+s_{1}(z) \exp \left(-i \delta x_{0}\right)\left|H_{m}^{(1)}\left(x_{0}\right)\right|\right) \\
& \quad-\frac{C_{1}(S-u+i \delta)}{v} \exp \left(S x_{0}-i \delta x_{0}\right)\left[1-\exp \left(2 S\left(x_{R}-x_{0}\right)\right)\right] \\
& \quad \times\left(H_{m}^{(2)}\left(x_{0}\right) Z(z)+s_{-1}(z) \exp \left(i \delta x_{0}\right)\left|H_{m}^{(1)}\left(x_{0}\right)\right|\right)
\end{aligned}
$$

and

$$
\begin{aligned}
& A\left(g_{A}+i\right) Z(z)\left[\exp \left(g_{A} x_{0}\right) H_{m}^{(1)}\left(x_{0}\right)-\exp \left(-g_{A} x_{0}\right) H_{m}^{(2)}\left(x_{0}\right)\right] \\
& =C_{1}\left[S+i \delta-(S-i \delta) \frac{S-u+i \delta}{S+u-i \delta} \exp \left(2 S\left(x_{R}-x_{0}\right)\right)\right] \\
& \quad \times \exp \left(S x_{0}+i \delta x_{0}\right)\left(H_{m}^{(1)}\left(x_{0}\right) Z(z)+s_{1}(z)\right. \\
& \left.\quad \times \exp \left(-i \delta x_{0}\right)\left|H_{m}^{(1)}\left(x_{0}\right)\right|\right)+i C_{1} \exp \left(S x_{0}+i \delta x_{0}\right)
\end{aligned}
$$

$$
\left\{\begin{array}{l}
A(x)=C_{1} \exp (S x+i \delta x)\left[1+\frac{S-u+i \delta}{S+u-i \delta} \exp \left(2 S\left(x_{R}-x\right)\right)\right] \\
B(x)=-\frac{C_{1}(S-u+i \delta)}{v} \exp (S x-i \delta x)\left[1-\exp \left(2 S\left(x_{R}-x\right)\right)\right] \\
E_{z}^{(m)}(x, z)=C_{1} \exp (S x+i \delta x)\left[1+\frac{S-u+i \delta}{S+u-i \delta} \exp \left(2 S\left(x_{R}-x\right)\right)\right]\left(H_{m}^{(1)}(x) Z(z)+s_{1}(z) \exp (-i \delta x)\left|H_{m}^{(1)}(x)\right|\right) \\
-\frac{C_{1}(S-u+i \delta)}{v} \exp (S x-i \delta x)\left[1-\exp \left(2 S\left(x_{R}-x\right)\right)\right]\left(H_{m}^{(2)}(x) Z(z)+s_{-1}(z) \exp (i \delta x)\left|H_{m}^{(1)}(x)\right|\right)
\end{array}\right.
$$




$$
\begin{aligned}
& \times\left[1+\frac{S-u+i \delta}{S+u-i \delta} \exp \left(2 S\left(x_{R}-x_{0}\right)\right)\right] \\
& \times\left(H_{m}^{(1)}\left(x_{0}\right) Z(z)-\delta s_{1}(z) \exp \left(-i \delta x_{0}\right)\left|H_{m}^{(1)}\left(x_{0}\right)\right|\right) \\
& -\frac{C_{1}(S-u+i \delta)}{v}\left[S-i \delta+(S+i \delta) \exp \left(2 S\left(x_{R}-x_{0}\right)\right)\right] \\
& \times \exp \left(S x_{0}-i \delta x_{0}\right)\left(H_{m}^{(2)}\left(x_{0}\right) Z(z)+s_{-1}(z)\right. \\
& \left.\times \exp \left(i \delta x_{0}\right)\left|H_{m}^{(1)}\left(x_{0}\right)\right|\right)+i \frac{C_{1}(S-u+i \delta)}{v} \\
& \times \exp \left(S x_{0}-i \delta x_{0}\right)\left[1-\exp \left(2 S\left(x_{R}-x_{0}\right)\right)\right] \\
& \times\left(H_{m}^{(2)}\left(x_{0}\right) Z(z)-\delta s_{-1}(z) \exp \left(i \delta x_{0}\right)\left|H_{m}^{(1)}\left(x_{0}\right)\right|\right)
\end{aligned}
$$

Multiplying both the sides of (31) and (32) by $Z(z)$, integrating them over $z$, and dividing (31) by (32) yields the following characteristic equation of the laser mode:

$$
\frac{\exp \left(2 g_{A} x_{0}\right) H_{m}^{(1)}\left(x_{0}\right)+H_{m}^{(2)}\left(x_{0}\right)}{\left(g_{A}+i\right)\left[\exp \left(2 g_{A} x_{0}\right) H_{m}^{(1)}\left(x_{0}\right)-H_{m}^{(2)}\left(x_{0}\right)\right]}=\frac{\xi}{\zeta}
$$

where

$$
\begin{aligned}
\xi= & {\left[1+\frac{S-u+i \delta}{S+u-i \delta} \exp \left(2 S\left(x_{R}-x_{0}\right)\right)\right] \exp \left(2 i \delta x_{0}\right) H_{m}^{(1)}\left(x_{0}\right) } \\
& -\frac{(S-u+i \delta)}{v}\left[1-\exp \left(2 S\left(x_{R}-x_{0}\right)\right)\right] H_{m}^{(2)}\left(x_{0}\right) \\
\zeta= & {\left[S+i \delta-(S-i \delta) \frac{S-u+i \delta}{S+u-i \delta} \exp \left(2 S\left(x_{R}-x_{0}\right)\right)\right] } \\
& \times \exp \left(2 i \delta x_{0}\right) H_{m}^{(1)}\left(x_{0}\right)+i\left[1+\frac{S-u+i \delta}{S+u-i \delta}\right. \\
& \left.\times \exp \left(2 S\left(x_{R}-x_{0}\right)\right)\right] \times \exp \left(2 i \delta x_{0}\right) H_{m}^{(1)}\left(x_{0}\right) \\
& -\frac{(S-u+i \delta)}{v}\left[S-i \delta+(S+i \delta) \exp \left(2 S\left(x_{R}-x_{0}\right)\right)\right] \\
& \times H_{m}^{(2)}\left(x_{0}\right)+i \frac{(S-u+i \delta)}{v}\left[1-\exp \left(2 S\left(x_{R}-x_{0}\right)\right)\right] \\
& \times H_{m}^{(2)}\left(x_{0}\right) .
\end{aligned}
$$

Note that $\int_{-\infty}^{+\infty} s_{ \pm 1}(z) Z(z) d z=0$ (recalling that $Z(z)$ is an eigenstate of vertical-mode equation, it can be shown by an argument similar to the orthogonality condition of the bound modes). Since (33) is a complex equation, its solution yields both the threshold gain $g_{A}$ and the frequency detuning $\delta$. It should be noted that in the calculation of the threshold gain levels, we consider only the loss stemming from the finite Bragg reflectivity and the vertical radiation. Other loss mechanisms, such as free carrier absorption or random scattering, are not included in the derivation.

The power loss in a circular Bragg microdisk laser is composed of two contributions - the coherently scattered, vertically emitted light comprising our useful signal, and the transverse loss due to the finite radial length of the Bragg reflectors

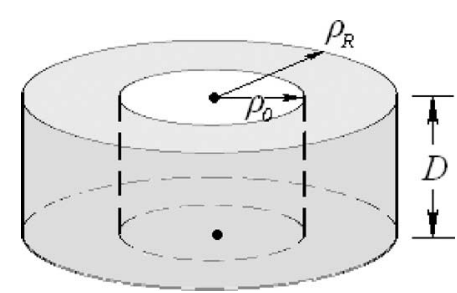

Fig. 2. Model for calculation of the normalized total optical loss.

(see Fig. 1). Using the total energy stored in the resonator

$$
\frac{1}{4} \iiint_{\substack{\text { within } \\ \text { resonator }}}\left(\varepsilon|\vec{E}|^{2}+\mu|\vec{H}|^{2}\right) d V
$$

and the outflow power through a surface

$$
\iint_{\text {surface }}(\vec{E} \times \vec{H}) \cdot d \vec{A}
$$

we can define the normalized total optical loss $L$ as

$$
\begin{aligned}
L= & \frac{\int_{0}^{2 \pi} \int_{\rho_{0}}^{\rho_{R}}|\Delta E(\rho, z=0)|^{2} \rho d \rho d \varphi}{\int_{0}^{D} \int_{0}^{2 \pi} \int_{0}^{\rho_{R}}|E(\rho, z)|^{2} \rho d \rho d \varphi d z} \\
& +\frac{\int_{0}^{D} \int_{0}^{2 \pi}\left|E\left(\rho=\rho_{R}, z\right)\right|^{2} \rho_{R} d \varphi d z}{\int_{0}^{D} \int_{0}^{2 \pi} \int_{0}^{\rho_{R}}|E(\rho, z)|^{2} \rho d \rho d \varphi d z} .
\end{aligned}
$$

The integration regions are shown in Fig. 2.

\section{Numerical Results AND the Optimization OF DESIGN PARAMETERS}

We assume a circular Bragg laser with a layer structure as described in [12], which was designed for $1.55-\mu \mathrm{m}$ laser emission. For simplicity, we approximate the complicated layer structure by an effective index profile comprising of five layers: 1 ) lower cladding, $n=1.54$; 2) first layer, $n=3.281$ and thickness of $60.5 \mathrm{~nm} ; 3)$ second layer (the active region), $n=3.4057$ and thickness of $129 \mathrm{~nm}$; 4) third layer, $n=3.281$ and thickness of $60.5 \mathrm{~nm}$, and 5) upper cladding, $n=1.54$. Numerical calculations of the mode profile and the effective index of the approximated layer structure indicate negligible deviations from those of the exact one. However, unlike the structure described in [12], we focus our analysis on the case of shallow gratings with an etch depth of $\sim 185 \mathrm{~nm}$. For this structure, the vertical mode profile $Z(z)$, the effective index $n_{\text {eff }}$ (4), and the Green's function (19) are numerically calculated.

We assume a rectangular grating profile with a Hankel-phased modulation (see [8])

$$
\Theta\left(\Phi\left[H_{m}^{(1)}(x)\right], \alpha\right)= \begin{cases}1, & \cos \left(\Phi\left[H_{m}^{(1)}(x)\right]\right) \geq \alpha \\ 0, & \cos \left(\Phi\left[H_{m}^{(1)}(x)\right]\right)<\alpha\end{cases}
$$

which can be expanded in Fourier series as

$$
\begin{aligned}
& \Theta\left(\Phi\left[H_{m}^{(1)}(x)\right], \alpha\right) \\
& \quad=\frac{\arccos \alpha}{\pi}+\frac{2}{\pi} \sum_{m=1}^{\infty} \frac{\sin (m \arccos \alpha)}{m} \cos \left(m \Phi\left[H_{m}^{(1)}(x)\right]\right)
\end{aligned}
$$




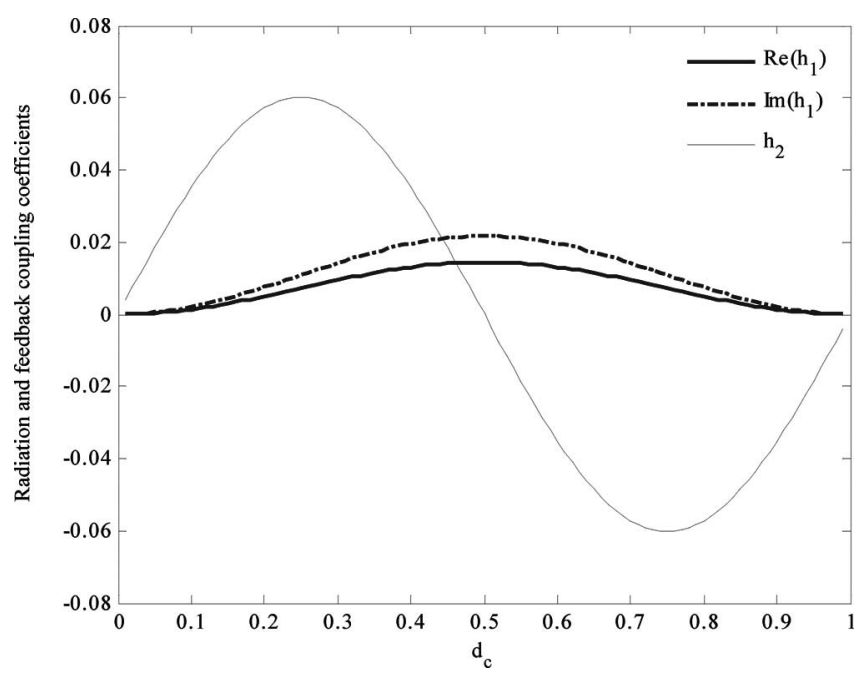

Fig. 3. Radiation coupling coefficient $h_{1}$ and the feedback coupling coefficient $h_{2}$ as a function of the duty cycle $d_{c}$ of the Hankel-phase-modulated rectangular gratings.

$$
\begin{aligned}
\approx & \frac{\arccos \alpha}{\pi}+\frac{2}{\pi}\left[\sin (\arccos \alpha) \cos \left(\Phi\left[H_{m}^{(1)}(x)\right]\right)\right. \\
& \left.+\frac{\sin (2 \arccos \alpha)}{2} \cos \left(2 \Phi\left[H_{m}^{(1)}(x)\right]\right)\right] \\
= & d_{c}+\frac{1}{2 \pi} \sin \left(2 \pi d_{c}\right)\left[\exp \left(-i 2 \Phi\left[H_{m}^{(1)}(x)\right]\right)\right. \\
& \left.+\exp \left(i 2 \Phi\left[H_{m}^{(1)}(x)\right]\right)\right]+\frac{1}{\pi} \sin \left(\pi d_{c}\right) \\
& \times\left[\exp \left(-i \Phi\left[H_{m}^{(1)}(x)\right]\right)+\exp \left(i \Phi\left[H_{m}^{(1)}(x)\right]\right)\right] .
\end{aligned}
$$

Note that the expansion yields the following coefficients:

$$
a_{2}=a_{-2}=\frac{\sin \left(2 \pi d_{c}\right)}{2 \pi}
$$

and

$$
a_{1}=a_{-1}=\frac{\sin \left(\pi d_{c}\right)}{\pi}
$$

where

$$
d_{c} \equiv \frac{\arccos \alpha}{\pi} \quad\left(-1<\alpha<1,0<d_{c}<1\right)
$$

is the duty cycle of the Hankel-phase-modulated rectangular gratings. It should be emphasized that the duty cycle has a significant role in determining the coupling coefficients $h_{1}$ and $h_{2}$. Fig. 3 depicts $h_{1}$ and $h_{2}$ as a function of $d_{c}$ for $m=0$. Note that in general, $h_{1}$ is a complex number as already pointed out in [15]. Moreover, it has a relatively large imaginary part, which plays an important role in the coupled-mode equations (24). For example, when $d_{c}=0.5$, even though $h_{2}$ approaches zero, $\nu$ does not vanish because of the contribution from $h_{1}$. Thus, despite the absence of $h_{2}$, there is still a feedback. Since the coupling strength between the outgoing and incoming waves is related to the modulus of $\nu$ [16], we expect a large feedback around the maximum of $|\nu|$. Therefore, a judicious choice is $d_{c}=0.25$ because $h_{2}$ is maximal while $\operatorname{Re}\left(h_{1}\right)$ is not small and, thus, we expect to achieve a large ratio of useful signal to power

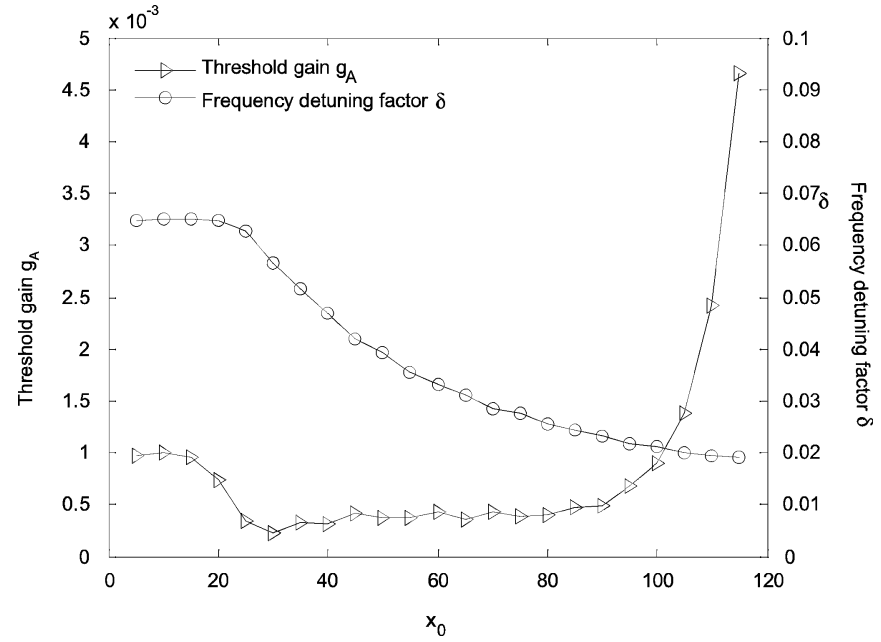

Fig. 4. Threshold gain $g_{A}$ and the frequency detuning factor $\delta$ of the first lasing modes of the circular Bragg microdisk lasers with different inner radii $x_{0}\left(d_{c}=0.25\right)$.

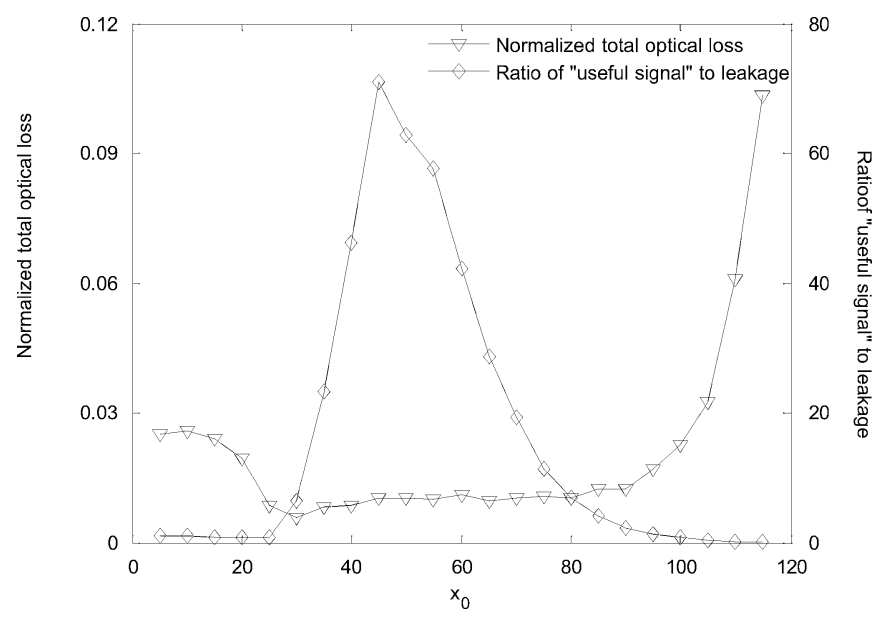

Fig. 5. Normalized total optical loss and the ratio of vertical radiation power ("useful signal") to periphery power leakage of the first lasing modes of the circular Bragg microdisk lasers with different inner radii $x_{0}\left(d_{c}=0.25\right)$.

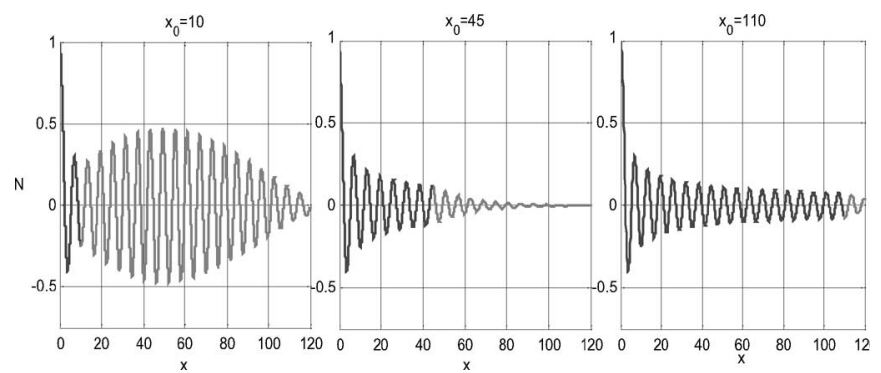

Fig. 6. Normalized radial profile of the in-plane electric fields $E(x)$ for designs with $x_{0}=10,45$, and $110\left(d_{c}=0.25\right)$. The field amplitude at the external boundary and hence, the power leakage are minimal for $x_{0}=45$.

leakage. Using (18), (20), (22), and (23), the coefficients are numerically calculated and found to be $h_{1}=0.0072+0.0108 i$ and $h_{2}=0.0601$.

It should be noted that we do not attempt to find the eigenmodes of a given laser structure, but rather search for the optimal laser structure exhibiting low lasing threshold and a large useful 


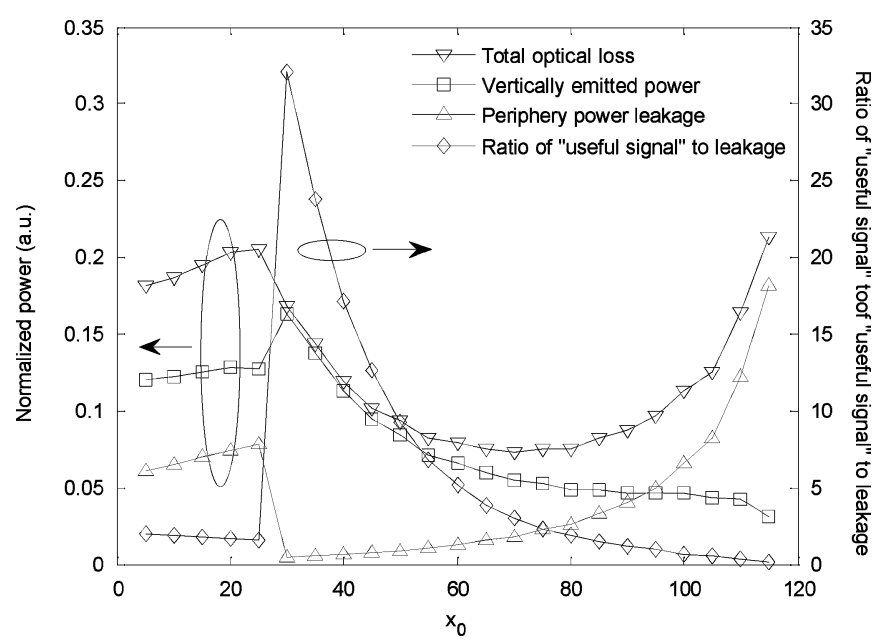

Fig. 7. Vertical radiation and leakage powers, their sum and ratio, for the first lasing modes of the circular Bragg microdisk lasers with different inner radii $x_{0}\left(d_{c}=0.5\right)$.

signal to power leakage ratio. In general, larger devices having more Bragg layers yield (arbitrarily) low threshold levels. Therefore, we predefine the overall size of the device, keeping the external boundary $x_{R}$ fixed and optimize the inner radius $x_{0}$. We assume a typical device with a diameter of $\sim$ $20 \mu \mathrm{m}\left(\rho_{R} \approx 10 \mu \mathrm{m}\right)$, so, $x_{R}=\beta \rho_{R}=2 \pi / \lambda_{0} \times n_{\mathrm{eff}} \times \rho_{R} \approx$ $2 \pi / 1.55 \times 2.83 \times 10 \approx 120$. Given a pair of $x_{0}$ and $x_{R}$, we put them into (33), solve for all the allowed pairs of $g_{A}$ and $\delta$, and then pick up amongst them the first lasing mode that has the smallest $g_{A}$. Fig. 4 shows the threshold gain $g_{A}$ and the frequency detuning factor $\delta$ of the first lasing mode of the circular Bragg microdisk laser as a function of the inner radius $x_{0}$. Fig. 5 depicts the normalized total optical loss and the ratio of useful signal to power leakage. Since the lasing threshold is directly related to the total loss of a resonator, by comparing Figs. 4 and 5, the threshold gain $g_{A}$ and the total optical loss $L$, though obtained in different ways, have a highly similar dependence on $x_{0}$. Note that for $x_{0}$ between 25 and 90, the threshold gain remains below $5 \times 10^{-4}$, which is an order of magnitude lower than that reported for conventional radial distributed feedback (DFB) lasers [17]. Fig. 5 also shows a peak of the ratio between the useful signal and the power leakage at $x_{0}=45$. The numerical results give that for $x_{0}=45$, the threshold gain $g_{A}$ of the first lasing mode is $4.16 \times 10^{-4}$, which is within the low-threshold regime (see Fig. 4), thus, making it the desired (optimal) design. Actually, the maximal ratio of the laser emission to the internal loss at the optimal point $x_{0}=45$ is largely due to the minimal power leakage at that point, as shown in Fig. 6, which displays the normalized radial profile of the in-plane electric fields for designs of different disk radii $x_{0}$.

Referring to Figs. 4 and 5, we note that the threshold gain level $g_{A}$ and the total optical loss $L$ remain low for $25 \leq x_{0} \leq 90$, but increase rapidly for smaller and larger $x_{0}$. Similar trends were found for devices employing different duty cycles. These trends stem from the presetting of the overall size of the resonator $x_{R}$. For large $x_{0}$, there are fewer Bragg gratings in the external reflector and, consequently, its reflectivity is reduced leading to more power leakage. On the other hand, shorter grating region also reduces the emission from the surface, thus creating a tradeoff between the two sources of loss-vertical radiation and the periphery power leakage. This tradeoff results in an optimal $x_{0}$ which minimizes the total optical loss and the threshold gain level. Fig. 7 depicts the vertical radiation power, the leakage power, their sum and ratio, for $d_{c}=0.5$. The duty cycle of one half was chosen to attain $h_{2}=0$ such that the comparable real and imaginary parts of $h_{1}$ account respectively for the radiation coupling and feedback coupling. Since we always pick up the first lasing mode as the one with the lowest threshold gain, the discontinuity at $x_{0}=25$ stems from a switch of choosing the first lasing mode (as can be confirmed from the plot of frequency detuning factors).

\section{CONCLUSION}

We derived the coupled-mode equations for mixed order gratings in 3-D cylindrical coordinates including the impact of vertical radiation, and applied them to the analysis of circular Bragg microdisk lasers. We obtained the characteristic equation, the threshold gain levels and the resonance frequency detuning factors. We also found a direct relation between the total optical loss and the threshold gain, offering a powerful method for optimally designing the device structure. Reducing the threshold gain while maximizing the ratio of the laser radiation to the internal loss, we suggested an optimal design for the circular Bragg microdisk lasers. The theoretically obtained threshold gain level is more than an order of magnitude lower than that reported for conventional (periodic) radial DFB lasers.

\section{ACKNOWLEDGMENT}

The authors would like to thank Dr. P. Chak and W. Mao for helpful discussions.

\section{REFERENCES}

[1] S. J. Choi, K. Djordjev, S. J. Choi, and P. D. Dapkus, "Microdisk lasers vertically coupled to output waveguides," IEEE Photon. Technol. Lett., vol. 15, no. 10, pp. 1330-1332, Oct. 2003.

[2] C. K. Madsen and J. H. Zhao, Optical Filter Design and Analysis: A Signal Processing Approach. New York: Wiley-Interscience, 1999.

[3] A. Yariv, "Critical coupling and its control in optical waveguide-ring resonator systems," IEEE Photon. Technol. Lett., vol. 14, no. 4, pp. 483485, Apr. 2002.

[4] V. Van, T. A. Ibrahim, K. Ritter, P. P. Absil, F. G. Johnson, R. Grover, J. Goldhar, and P.-T. Ho, "All-optical nonlinear switching in GaAsAlGaAs microring resonators," IEEE Photon. Technol. Lett., vol. 14 no. 1, pp. 74-76, Jan. 2002.

[5] S. M. Spillane, T. J. Kippenberg, K. J. Vahala, K. W. Goh, E. Wilcut, and H. J. Kimble, "Ultrahigh-Q toroidal microresonators for cavity quantum electrodynamics," Phys. Rev. Lett. A, vol. 71, no. 1, p. 013817, Jan. 2005.

[6] R. W. Boyd and J. E. Heebner, "Sensitive disk resonator photonic biosensor," Appl. Opt., vol. 40, no. 31, pp. 5742-5747, Nov. 2001.

[7] E. A. J. Marcatili, "Bends in optical dielectric guides," AT\&T Tech. J., vol. 48, pp. 2103-2132, Sep. 1969.

[8] J. Scheuer and A. Yariv, "Coupled-waves approach to the design and analysis of Bragg and photonic crystal annual resonators," IEEE J. Quantum Electron., vol. 39, no. 12, pp. 1555-1562, Dec. 2003.

[9] —-, "Annular Bragg defect mode resonators," J. Opt. Soc. Am. B vol. 20, no. 11, pp. 2285-2291, Nov. 2003.

[10] M. Meier, A. Mekis, A. Dodabalapur, A. Timko, R. E. Slusher J. D. Joannopoulos, and O. Nalamasu, "Laser action from two-dimensional distributed feedback in photonic crystals," Appl. Phys. Lett., vol. 74, no. 1 pp. 7-9, Jan. 1999. 
[11] J. Scheuer, W. M. J. Green, G. A. DeRose, and A. Yariv, "Lasing from a circular Bragg nanocavity with an ultrasmall modal volume," Appl. Phys. Lett., vol. 86, no. 25, p. 251101, Jun. 2005.

[12] — , "InGaAsP annular Bragg lasers: Theory, applications, and modal properties," IEEE J. Sel. Topics. Quantum Electron., vol. 11, no. 2, pp. 476-484, Mar./Apr. 2005.

[13] , "Low-threshold two-dimensional annular Bragg lasers," Opt. Lett., vol. 29, no. 22, pp. 2641-2643, Nov. 2004.

[14] A. Yariv, Optical Electronics in Modern Communications, 5th ed. New York: Oxford Univ. Press, 1997, p. 501.

[15] H. Wenzel, R. Guther, A. M. Shams-Zadeh Amiri, and P. Bienstman, "A comparative study of higher order Bragg gratings: Coupled-mode theory versus mode expansion modeling," IEEE J. Quantum Electron., vol. 42, no. 1, pp. 64-70, Jan. 2006

[16] A. Yariv, Optical Electronics in Modern Communications, 5th ed. New York: Oxford Univ. Press, 1997, ch. 16

[17] P. L. Greene and D. G. Hall, "Effects of radiation on circular-grating DFB lasers-Part I: Coupled-mode equations," IEEE J. Quantum Electron., vol. 37, no. 3, pp. 353-364, Mar. 2001.

Xiankai Sun was born in Wuhan, China, in 1982. He received the B.S. degree (with honors) in physics from the University of Science and Technology of China, Hefei, China, in 2004, and the M.S. degree in applied physics from the California Institute of Technology (Caltech), Pasadena, in 2006, where he is currently working toward the Ph.D. degree in applied physics.

His current research interests include theoretical and experimental study of the circular Bragg grating resonator lasers, photonic crystal resonators, and other nanophotonic devices.

Jacob Scheuer (S'99-M'01-SM'06) received the B.Sc. degree (summa cum laude) in electrical engineering and in physics, and the Ph.D. degree in electrical engineering from Technion-Israel Institute of Technology, Haifa, Israel, in 1993 and 2001, respectively.

He was a Chief Designer with Lambda Crossing-an optical component startup specializing in microring resonators for two years. Then, he joined the Center for the Physics of Information and the Department of Applied Physics, the California Institute of Technology, Pasadena, as a Research Associate. Currently, he is a Senior Lecturer with the School of Electrical Engineering, Tel-Aviv University, Ramat-Aviv, Tel-Aviv, Israel. His research interests include nanophotonics, polymer optics, slow light, and secure communications.

Dr. Scheuer is a member of the Optical Society of America.
Amnon Yariv (S'56-M'59-F'70-LF'95) received the B.S., M.S., and Ph.D. degrees in electrical engineering from the University of California, Berkeley, in 1954, 1956, and 1958, respectively.

In 1959, he was with Bell Telephone Laboratories, Murray Hill, NJ. In 1964 he joined the California Institute of Technology (Caltech), Pasadena, as an Associate Professor of Electrical Engineering, becoming a Professor in 1996. In 1980, he became the Thomas G. Myers Professor of Electrical Engineering and Applied Physics. In 1996, he became the Martin and Eileen Summerfield Professor of Applied Physics and Electrical Engineering. He participated in the discovery of several early solid-state laser systems; in the original formulation of the theory of nonlinear quantum optics; in proposing and explaining modelocked ultrashort-pulse lasers, GaAs optoelectronics, semiconductor-based integrated optics technology, phase conjugate optics, and semiconductor-distributed feedback laser. His current research interests include nonlinear optics, semiconductor lasers, and wavelength-division multiplexing (WDM) filters, especially the monolithic integration of transistors, injection lasers, ultrafast $(<1 \mathrm{ps})$ semiconductor devices and phenomena, and the propagation of optical signals in fibers, including WDM. He is the author of several books on quantum electronics, optics, and quantum mechanics.

Dr. Yariv received the 1980 Quantum Electronics Award of the IEEE, the 1985 University of Pennsylvania Pender Award, the 1986 Optical Society of America Ives Medal, the 1992 Harvey Prize (shared with M. Gorbachev), and the 1998 Esther Beller Medal of the Optical Society of America. He is a Founder and Chairman-of-the-Board of ORTEL Corporation (acquired by Lucent Technologies), and a Founder and Board Member of Arroyo Optics Inc. He is a member of the American Physical Society, the Phi Beta Kappa, the American Academy of Arts and Sciences, the National Academy of Engineering, the National Academy of Sciences, and a Fellow of the Optical Society of America. 